EESTI NSV TEADUSTE AKADEEMIA TOIMETISED.

FOUSIKA * MATEMAATIKA

ИЗВЕСТИЯ АКАДЕМИИ НАУК ЭСТОНСКОИ ССР. ФИЗИКА * МАТЕМАТИКА

PROCEEDINGS OF THE ACADEMY OF SCIENCES OF THE ESTONIAN SSR.

PHYSICS * MATHEMATICS

$1985,34,2$

удК $534.2+577.31$

Ю. ЭНГЕЛЬБРЕХТ, Т. ТОБИАС

\title{
ОБРАТНАЯ ЗАДАЧА ДЛЯ НЕЛИНЕЙНЫХ ВОЛН В АКТИВНОЙ СРЕДЕ
}

\author{
(Представил Н. Алумяэ)
}

1. Некоторые задачи распространения нелинейных волн в активной среде приводят к уединенным волнам $\left[{ }^{1,2}\right]$, имеющим определенные стационарные профили. С математической точки зрения это свидетельствует об автомодельности процесса, с физической точки зрения - об уравновешивании ряда физических эффектов, таких как нелинейность, диссипация, приток энергии. В физических экспериментах измерение стационарных профилей связано с меньшими трудностями по сравнению с измерением изменяющихся во времени и в пространстве профилей. Поэтому для решения обратной задачи восстановления коэффициентов начального уравнения, которые прямо связаны с физическими параметрами процесса, целесообразно использовать стационарные профили.

Далее рассматривается задача восстановления коэффициентов математической модели, описывающей распространение импульсов в нервном волокне. Для описания уединенной волны в нем предложена гиперболическая модель $\left[{ }^{3}\right]$, согласно которой волновой процесс описан эволюционным уравнением [ $\left.{ }^{4}\right]$

$$
\frac{\partial^{2} z}{\partial \xi \partial x}+\left(C_{1}-C_{2} z+a C_{2} z^{2}\right) \frac{\partial z}{\partial \xi}+\Theta C_{3} z=0
$$

где $C_{1}, C_{2}, C_{3}, a, \Theta$ - положительные постоянные. Переменной $\xi$ измеряется расстояние от псевдофронта $\xi=c_{0} t-x=0$, где $c_{0}-$ скорость волны без учета притока энергии, коэффициент $a$ связан с равновесным потенциалом, коэффициент $\Theta-$ с действительной скоростью процесса. После введения автомодельной переменной $\eta=x+\Theta \xi$ из (1) вытекает уравнение стационарной волны

$$
z^{\prime \prime}+\left(C_{1}-C_{2} z+a C_{2} z^{2}\right) z^{\prime}+C_{3} z=0,
$$

которое решается при условиях

$$
z(0)=0, \quad z^{\prime}(0)=C_{4} .
$$

Физическая сторона процесса следующая: уравнение (1) описывает эволюцию начального воздействия $z(0, \xi)=z_{0}(\xi)$ к уединенной волне (если это допущено физическими условиями [ $\left.\left.{ }^{4}\right]\right)$, а уравнение $(2)-$ стационарный профиль этой волны, когда физические эффекты, обусловливающие искажение профиля, уже уравновешены. В этом случае естественно задавать начальные условия (3), исходя из равновесного состояния. Процесс установления профиля изображен на рис. 1 , где приведены решение уравнения (1) при $C_{1}=3, C_{2}=3, C_{3}=1, a=0,1, \Theta=2,6$ (сплошная кривая ) на расстоянии $x=1,0$ см и решение уравнения (2) при 
$C_{4}=5,0$ (прерывистая кривая), а также начальное воздействие для уравнения (1) (штрих-пунктир).

Ставим следующую задачу: найти коэффициенты $C_{i}$ уравнения (2) и начального условия (3), если задано $z(\eta) \equiv f(\eta), \quad \eta \in[0, \varepsilon]$. На практике $f(\eta)$ представляет собой экспериментально определенную функцию.

2. Исследуем единственность задачи. Предположим, что задача имеет еще другое решение, т. е. выполняется

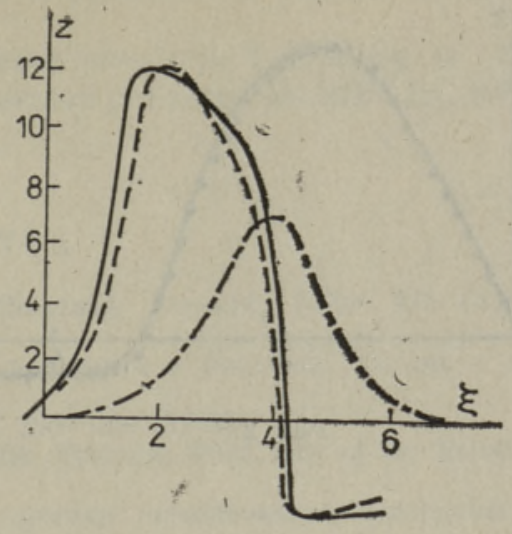

$$
\begin{aligned}
& z^{\prime \prime}+\left(d_{1}-d_{2} z+a d_{2} z^{2}\right) z^{\prime}+d_{3} z=0, \\
& z(0)=0, \quad z^{\prime}(0)=d_{4}, \quad \eta \in[0, \varepsilon],
\end{aligned}
$$

где $d_{i}$ - постоянные. Так как $z(\eta)$ задано, то $C_{4}=d_{4}=f^{\prime}(0)$. Вычитая уравнение (2) из (4) получим

$$
\begin{aligned}
& \left(\alpha_{1}-\alpha_{2} z+a_{\alpha_{2}} z^{2}\right) z^{\prime}+\alpha_{3} z=0, \\
& z(0)=0, \quad z^{\prime}(0)=C_{4} ; \quad \alpha_{i}=C_{i}-d_{i} .
\end{aligned}
$$

Так как $z(0)=0$, то $\alpha_{1} C_{4}=0$, следовательно, $\alpha_{1}=0$, и из (5) вытекает

$$
\left(-\alpha_{2}+a \alpha_{2} z\right) z^{\prime}+\alpha_{3}=0 .
$$

Пусть $\alpha_{3}=0$, тогда $\left(-\alpha_{2}+a \alpha_{2} z\right) z^{\prime}=0$. Это выполняется лишь в случае, когда $z \equiv$ const, что не удовлетворяет условиям задачи. Поэтому $\alpha_{3} \neq 0$. Деля (6) на $\alpha_{3}$ и интегрируя, получим

$$
-\frac{\alpha_{2}}{\alpha_{3}} z+\frac{a \alpha_{2}}{2 \alpha_{3}} z^{2}=-\eta+C, \quad C=\text { const. }
$$

Так как $z(0)=0$, то $C=0$, и в итоге получим

$$
z=a^{-1}\left(1-\left(1-2 a \alpha_{2}^{-1} \alpha_{3} \eta\right)^{1 / 2}\right),
$$

где знак минус выбран на основе начального условия. Покажем, что при любом $\beta$ функция $z=1-(1-\beta \eta)^{1 / 2}$ не может удовлетворять уравнению (2) на отрезке $[0, \varepsilon], \varepsilon>0$. Прямой подстановкой получим

$$
\begin{gathered}
\beta^{2}+2 \beta\left(C_{1}-C_{2}+a C_{2}\right)(1-\beta \eta)+2\left(\beta C_{2}-2 \alpha \beta C_{2}+2 C_{3}\right)(1-\beta \eta)^{3 / 2}+ \\
+2\left(a \beta C_{2}-2 C_{3}\right)(1-\beta \eta)^{2}=0 .
\end{gathered}
$$

Определитель Вронского для функций $1,1-\beta \eta,(1-\beta \eta)^{3 / 2}$ и $(1-\beta \eta)^{2}$ равен $W=-3 / 4 \beta^{6}(1-\beta \eta)^{-3 / 2} \neq 0$, следовательно, равенство (8) не выполняется. В итоге мы доказали, что решение $z(\eta), \eta \in[0, \varepsilon]$ определяет параметры $C_{1}, C_{2}, C_{3}$ и $C_{4}$ однозначно при любом $\varepsilon>0$.

3. Обозначим решение уравнения (2) через $z(\eta)=z(\eta, C)$, где $C=$ $=\left(C_{1}, C_{2}, C_{3}, C_{4}\right)$. На практике решение известно в дискретных точках, т. е. известны измерения $z_{i}=z\left(\eta_{i}, C\right), i=1, \ldots, N$. Поставим следующую задачу: определить $C$ так, чтобы

$$
J(C)=\sum_{i=1}^{N}\left[z\left(\eta_{i}, C\right)-z_{i}\right]^{2} \rightarrow \min .
$$




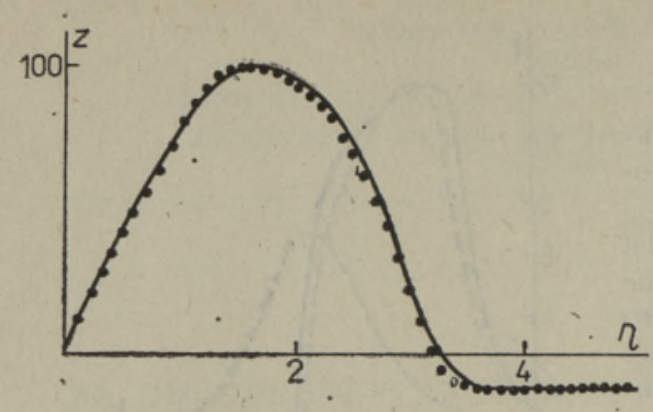

Рис. 2.

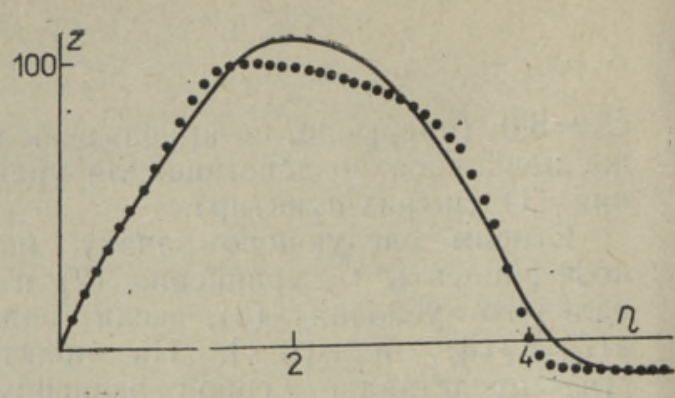

Рис. 3.

Опишем коротко градиентный метод решения поставленной задачи. Обозначим $u_{k}(\eta, C)=\partial z(\eta, C) / \partial C_{k}$. Тогда

$$
\frac{\partial J}{\partial C_{k}}=2 \sum_{i=1}^{N}\left[z\left(\eta_{i}, C\right)-z_{i}\right] u_{k}\left(\eta_{i}, C\right) \text {. }
$$

Построим последовательность $C^{n}$ приближений по правилу

$$
C^{n+1}=C^{n}-t_{n} \operatorname{grad} J\left(C^{n}\right),
$$

где $t_{n}$ - длина градиентного шага. Легко вывести уравнения для функций $u_{k}(\eta, C)$

$$
\begin{gathered}
u_{k}^{\prime \prime}+\left(C_{1}-C_{2} z+a C_{2} z^{2}\right) u_{k}^{\prime}+C_{3} u_{k}+\left(-C_{2}+2 a C_{2} z\right) z^{\prime} u_{k}=g_{k}, \\
u_{k}(0)=0, \quad u_{k}^{\prime}(0)=0, \quad k=1,2,3, \quad u_{4}(0)=0, \quad u_{4}^{\prime}(0)=1,
\end{gathered}
$$

где $g_{1}=-z^{\prime}, g_{2}=z z^{\prime}-a z^{2} z^{\prime}, g_{3}=-z, g_{4}=0$. Можно также воспользоваться алгоритмами без производных, описанными в $\left[{ }^{5}\right]$.

В нижеследующих численных расчетах использовалась готовая программа определения неизвестных коэффициентов дифференциальных уравнений, имеющаяся в пакете BMDP $\left[{ }^{6}\right]$.

Задача 1. В качестве заданной $f(\eta)$ выбрано решение уравнения (2) при $C_{1}=4,615, C_{2}=0,2115, C_{3}=1, C_{4}=140, a=1 / 110$. Ищутся коэффициенты уравнения (2) при $a=1 / 130$, последний с физической точки зрения лучше определяет равновесный потенциал $(-65$ мВ). Результаты расчета представлены на рис. 2 , где точки соответствуют значениям заданной функции при $N=50$, а сплошная линия - решению обратной задачи. Искомые коэффициенты получаются следующими: $C_{1}=3,531$, $C_{2}=0,128, C_{3}=0,945, C_{4}=142$. Остаточная сумма (минимальное значение функции $J(C)$ ) равна 185,35 . Вариация начальных данных, как и следовало ожидать, не влияла на окончательный результат.

Задача 2. Заданная функция $f(\eta)$ выбрана исходя из предполагаемых значений максимальной $(\sim 100$ мВ) и минимальной $(\sim-10$ мВ) амплитуды и показана на рис. 3 точками (всего $N=50$ точек). Начальные данные выбраны из решения задачи 1: $C_{1}=3,531, C_{2}=0,128, C_{3}=0,945$, $C_{4}=120 \quad(a=1 / 130)$. Результат расчета изображен на рис. 3 сплошной линией, искомые значения параметров следующие: $C_{1}=2,61, C_{2}=0,091$, $C_{3}=0,568, C_{4}=121$. Остаточная сумма (минимальное значение функции $J(C))$ равна 1969. Вполне ясно, что заданная функция неудовлетворительно описывается используемой моделью, т. е. решением уравнения (2).

Недостатком указанных алгоритмов является невозможность качест- 
венной проверки существованйя порога и уровня насыщения (максимальной возможной амплитуды).

В заключение авторы выражают признательность Т. Тийтсу за оказанную помощь при осуществлении численных расчетов на ЭВМ ЕС 1052.

\section{ЛИТЕ РАТ У РА}

1. Автомодельные процессы в системах с диффузией. Горький, ИПФ АН СССР, 1981.

2. Марри Дж. Нелинейные дифференциальные уравнения в биологии. Лекции о моделях. М., «Мир», 1983.

3. Engelbrecht, J. Proc. Roy. Soc. London, A 375, 195-209 (1981).

4. Engelbrecht, $J$. Nonlinear waves in active media. Preprint, Acad. Sci. of the Estonian SSR, Tallinn, 1983.

5. Успенский A. Б. В кн.: Математические методы планирования эксперимента. Новосибирск, «Наука», 1981, 199-242.

6. BMDP, Biomedical Computer Programs, Ed. by W. Dixon. University of California Press, 1979.

Институт кибернетики

Академии наук Эстонской ССР
Поступила в редакцию 31/I 1984

\section{J. ENGELBRECHT, T. TOBIAS}

\section{AKTIIVSES KESKKONNAS LEVIVATE MITTELINEAARSETE LAINETE POORDULESANNE}

Aktiivses keskkonnas on võimalik statsionaarse profiiliga lainete formeerumine, kui energia juurdevool on tasakaalustatud dissipatsiooni- ja dispersiooniefektiga. Statsionaarse profiiliga laine kirjeldamiseks piisab harilikust diferentsiaalvoorrandist, mis tunduvalt hōlbustab ülesande lahendamist. Kōnealune pöördülesanne kujutab selle vōrrandi koefitsientide leidmist eksperimentaalselt määratud laineprofiili kuju järgi. Näitena on vaadeldud närviimpulsi levi kirjeldava mittelineaarse voorrandi koefitsientide leidmist ning tõestatud selle ülesande ühesus. Ulesanne on lahendatud gradientmeetodil ja toodud numbrilised näited.

\section{J. ENGELBRECHT, T. TOBIAS}

\section{AN INVERSE PROBLEM FOR NONLINEAR WAVES IN AN ACTIVE MEDIUM}

In several cases a solitary wave of a constant profile may form in an active medium, provided the energy influx and the dissipative and/or dispersion effects are balanced. Although the wave propagation process is generally governed by a partial differential equation, the constant profile is governed by a certain ordinary differential equation that makes a suitable starting point for solving the inverse problem. Here the wave process in a nerve fibre is studied and the corresponding inverse problem for the ordinary differential equation (2) governing the propagation of a stationary wave is stated. The formation of the stationary wave is demonstrated by solving the evolution equation (1). The inverse problem includes the determination of the coefficients of the ordinary differential equation on the basis of the known solution resulting from an experiment. The uniqueness of the corresponding inverse problem is established. Further the gradient method is proposed in order to get numerical solution. The numerical results obtained, using the Biomedical Computer Programs $\left[{ }^{6}\right]$, show a rather good convergence towards the established solitary wave profile. 